\title{
Integrais múltiplas na Engenharia: uma primeira revisão bibliográfica
}

\author{
GALVINA MARIA DE SOUZA ${ }^{1}$ \\ GABRIEL LOUREIRO DE LIMA ${ }^{2}$
}

\begin{abstract}
Resumo
Esse trabalho apresenta uma primeira revisão bibliográfica realizada acerca de teses de doutorado, produzidas entre 2000 e 2018, tendo como foco de estudo os processos de ensino e de aprendizagem de Integrais Múltiplas em cursos de Engenharia. São analisados dois trabalhos, destacando, inicialmente, título, autor, instituição e ano de publicação, para, posteriormente, analisarmos as questões ou objetivos das investigações, as abordagens teóricas às quais permitiram a análise dos dados encontrados, aspectos relacionados aos procedimentos metodológicos, bem como os principais resultados encontrados. Também buscamos identificar possiveis aspectos da Matemática no Contexto das Ciências - MCC, presentes, mesmo que implicitamente nessas pesquisas. Os resultados apontaram para a necessidade de realização de novas pesquisas, com olhares diferentes para as Integrais Múltiplas fora do contexto de problemas de cálculos de medidas de áreas de superfícies e de volumes de sólidos.
\end{abstract}

Palavras-Chave: Integrais Múltiplas; Engenharia; Revisão Bibliográfica; A Matemática no Contexto das Ciências.

\begin{abstract}
This work presents an initial literature review we conducted about PhD theses produced between 2000 and 2018, focusing on the study of teaching and learning processes of Multiple Integrals in Engineering courses. Two papers are discussed, highlighting, initially, title, author and year of publication, to, subsequently, analyze the issues or objectives of the research, the theoretical approaches which allowed the analysis of the data found, aspects related to the methodological procedures, and also the main results found. We also seek to identify possible aspects of Mathematics in the context of science - MCC, which are present even with implicitly in these recearchs. The results pointed to the need for further research, with different looks for the Multiple Integrals out of the context of measurement problems of surface areas and solids volumes.
\end{abstract}

Keywords: Multiple Integrals. Engineering. Bibliographical Review. Mathematics in the context of Science.

\section{Introdução}

Algumas pesquisas apresentam propostas e alternativas para se trabalhar com o Cálculo de Várias Variáveis, entretanto, após realizar um processo de levantamento e descrição de informações acerca das pesquisas produzidas quando o objeto matemático estudado são as Integrais Múltiplas, constatamos que existem poucas pesquisas neste recorte, principalmente teses de doutorado.

\footnotetext{
1 Pontifícia Universidade Católica de São Paulo. PEPG em Educação Matemática - e-mail: galvina04@yahoo.com.br.

2 Pontifícia Universidade Católica de São Paulo. PEPG em Educação Matemática - e-mail: gllima@pucsp.br.
} 
Em 2017, Lima, Bianchini e Gomes publicaram um mapeamento das pesquisas realizadas no âmbito do grupo de pesquisa GT04 - Ensino Superior - da SBEM (Sociedade Brasileira de Educação Matemática), que corroboram com os resultados de nosso levantamento, pois ao retratarem "investigações relativas às temáticas Cálculo Diferencial e Integral, Análise Matemática e intersecções desses campos de conhecimento" (p. 317), observaram "um número reduzido de investigações referentes aos conceitos de Cálculo associados às funções reais de duas ou mais variáveis reais," (p. 323) destacando:

[...] a ausência de estudos contemplando os processos de ensino e de aprendizagem de alguns tópicos importantes para a área de Cálculo Diferencial e Integral e suas aplicações, como, por exemplo, equações diferenciais, funções vetoriais, integrais duplas e triplas, derivada direcional e vetor gradiente, mudança de variável para integrais duplas ou triplas, integrais de linha, sequências, séries, etc. (LIMA, BIANCHINI e GOMES, 2017, p. 323).

Neste texto analisaremos as duas únicas teses de doutorado encontradas em nosso levantamento realizado, principalmente, no Banco de Teses da CAPES, na Biblioteca Digital Brasileira de Teses e Dissertações e no Google Acadêmico, tendo como palavras chave: Integrais Múltiplas, Funções de Várias Variáveis e Ensino de Integrais, considerando como critério de inclusão o período de 2000 a 2018 e o objeto matemático Integrais Múltiplas, com interesse de evidenciar o que revelam as pesquisas em Educação Matemática em relação aos processos de ensino e de aprendizagem deste objeto em cursos de Engenharia. Para isso, criamos uma organização para os dados, destacando, inicialmente, título, autor, instituição e ano de publicação, para, posteriormente, analisarmos as questões ou objetivos das investigações, as abordagens teóricas que fundamentaram a análise dos dados obtidos pelos pesquisadores, aspectos relacionados aos procedimentos metodológicos, bem como os principais resultados encontrados. Buscamos, finalmente, identificar possíveis aspectos consonantes com preceitos da teoria Matemática no Contexto das Ciências - MCC - presentes nessas pesquisas, de maneira explícita ou implícita.

Convém salientar que a MCC, desenvolvida pela pesquisadora Patricia Camarena, é o aporte teórico de nossa pesquisa de doutorado em andamento, e que tem como objetivo construir, implementar e avaliar eventos contextualizados para o ensino de Integrais 
Múltiplas em cursos de Engenharia. Tais eventos, segundo Camarena (2013), são problemas ou projetos elaborados com o objetivo de integrar disciplinas matemáticas e não matemáticas, constituindo-se, portanto, em ferramentas para o desenvolvimento de um trabalho interdisciplinar no ambiente de aprendizagem. Não é nosso objetivo nesse artigo apresentar detalhes referentes à MCC, mas salientamos que, dentre seus principais preceitos estão: o desenvolvimento de currículos de matemática para a engenharia que possibilitem ao estudante, durante sua graduação, o acesso a uma matemática voltada para o âmbito social de sua futura profissão e que o permita adquirir os elementos e ferramentas matemáticas necessárias às disciplinas específicas de seu curso de graduação; e a preocupação em trabalhar os conceitos matemáticos de maneira vinculada às suas aplicações nas disciplinas específicas e profissionalizantes ou ao contexto do cotidiano profissional do engenheiro. Para maiores detalhes acerca da MCC, sugere-se consultar Camarena (1984; 1995) e Camarena e Allier (2012).

Passamos então, efetivamente, a apresentar as duas teses de doutorado, realizadas entre 2000 e 2018, a que tivemos acesso até o presente momento da revisão bibliográfica de nossa pesquisa e que se referem aos processos de ensino e de aprendizagem de Integrais Múltiplas.

\section{Uma revisão bibliográfica inicial acerca dos processos de ensino e de aprendizagem de Integrais Múltiplas}

A investigação de Henriques (2006), realizada na França e intitulada L'enseignement et l'apprentissage des intégrales multiples: Analyse didactique intégrant l'usage du logiciel Maple, teve como sujeitos de pesquisa estudantes da Universidade Estadual de Santa Cruz - UESC, em Ilhéus-BA, e das classes preparatórias tecnológicas francesas - que preparam os estudantes para cursos superiores de tecnologia. Seu principal objetivo foi compreender as dificuldades apresentadas pelos estudantes durante os processos de ensino e de aprendizagem das Integrais Múltiplas utilizadas em problemas que envolvem o cálculo de medidas de volumes de sólidos, bem como, estudar como a utilização do software Maple pode ajudá-los a superar tais dificuldades. Dessa forma, sua abordagem a partir do Maple buscou favorecer as interações entre representações analíticas e gráficas dos sólidos estudados.

Com o objetivo de fundamentar, compreender e interpretar os fenômenos que envolvem o ensino e a aprendizagem dessas integrais, o autor apoiou-se na Teoria dos 
Registros de Representação Semiótica, proposta por Duval (1993), na Teoria Antropológica do Didático proposta por Chevallard (1999) e na Teoria da Instrumentação proposta por Rabardel (1995), por entender que cada uma dessas teorias e as interrelações entre elas trariam contribuições fundamentais para sua investigação.

A partir dos Registros de Representação Semiótica o autor pôde definir o que chamou de representação gráfica e representação analítica de um sólido, interpretar essas representações e as interações entre elas, entre os objetos manipulados pelo Maple e os objetos manipulados pelos estudantes utilizando o Maple; para as questões relacionadas à dimensão institucional utilizou aspectos abordados pela Teoria Antropológica do Didático e; para as questões referentes a dimensão instrumental da aprendizagem em ambientes computacionais - a aprendizagem do uso de ferramentas tecnológicas - fundamentou-se em pesquisas sobre ergonomia cognitiva encontradas na Teoria da Instrumentação.

$\mathrm{O}$ autor dividiu o seu trabalho em dois grupos, sendo que o primeiro analisou programas institucionais e manuais de referência das instituições nas quais foi desenvolvida a sua pesquisa, para entender como está organizada a matemática quando o objeto em estudo são as Integrais Múltiplas e como esses conteúdos são abordados nesses documentos, para evidenciar as práticas realizadas com a utilização do Maple para esse objeto matemático, e, por fim, para compreender como as representações analíticas e gráficas dos sólidos são apresentadas e a forma como esses dois tipos de representação interagem em situações de cálculo de volumes a partir desse objeto matemático. (HENRIQUES, 2006).

No segundo grupo, o autor realizou um estudo sobre o Maple, com o objetivo de identificar as ferramentas disponíveis nesse software capazes de construir representações gráficas dos sólidos gerados a partir de regiões utilizando as Integrais Múltiplas, além de entender como os estudantes se apropriam dessas ferramentas durante essas construções aplicadas a problemas de cálculo de medidas de volume, bem como, identificar as dificuldades que poderiam ser geradas pelo uso desse software.

Assim, na UESC foram vistos os documentos dos cursos de licenciatura e bacharelado em matemática que estavam em vigor a partir de 1999, nos quais o autor constatou que seu objeto em estudo estava alocado na matéria: Cálculo Diferencial e Integral, dividida em quatro 
disciplinas, Cálculo I a IV, estando presente nos dois primeiros anos de formação do curso de licenciatura em Matemática e nos três primeiros anos do curso de bacharelado em Matemática, bem como, que esse objeto aparece articulado às disciplinas Geometria Analítica e Geometria Descritiva, principalmente no que se refere ao estudo das funções de uma e várias variáveis e das representações gráficas dessas funções no plano e no espaço, além de se fazerem presentes também, nas disciplinas: Cálculo Numérico, Cálculo Avançado e Análise Real. Seu estudo contempla as integrais duplas, medidas de área de regiões planas e de volume de sólidos, integrais duplas em coordenadas polares, medida de área de superfícies tridimensionais, integrais triplas, coordenadas cilíndricas e esféricas, mudança de variáveis e cálculo vetorial: campos vetoriais, integrais curvilíneas, independência de caminhos, teorema de Green, teorema de Gauss e teorema de Stokes.

Esse objeto matemático foi alocado em três nichos ecológicos ${ }^{3}$ : o nicho estrutural, pois aparece a partir do estudo das funções de uma variável com as integrais simples até o estudo das funções de várias variáveis - nicho da análise matemática; o nicho geométrico, pois as Integrais Múltiplas são utilizadas para cálculo de medidas de áreas de superfícies e de volumes de sólidos gerados a partir dessas superfícies, utilizando-se das técnicas de representação gráfica e do raciocínio geométrico - nicho interpretativo; e o nicho aplicativo, por serem mobilizadas no trabalho com conteúdos aplicados na Física, principalmente em centros de massa e momentos de inércia. Já com os documentos analisados na instituição francesa, observou-se que as Integrais Múltiplas também aparecem alocadas nos nichos da análise estrutural, geométrica com abordagem somente para Integrais Duplas, e aplicativa, também com aplicações em física, tendo como objetos de estudo basicamente, os mesmos tópicos abordados na instituição brasileira com alguma diferença apenas na forma de organização das disciplinas.

Quanto ao Maple observou-se que, na França, é usado nas representações geométricas de curvas e superfícies. Não foram encontrados registros do uso desse software como ferramenta para construir representações geométricas de sólidos associados ao cálculo de volumes, na época da realização da investigação.

Também foram analisados livros didáticos, com o objetivo de identificar os principais elementos da aprendizagem de Superfícies e das Integrais Múltiplas, destacando dois livros de um mesmo autor com tradução do inglês para o português e do inglês para o francês,

\footnotetext{
${ }^{3}$ Nicho ecológico como definido em Chevallard (1992), ou seja, como sendo o lugar funcional ocupado
} 
escolhidos por aparecerem em programas institucionais e manuais de referência das instituições estudadas. Ambos apresentavam uma estrutura bem similar. Neles, o autor constatou que para o estudo de funções de uma variável, a abordagem algébrica desses objetos é bem estabelecida e a abordagem geométrica aparece, com maior ênfase, para calcular medidas de áreas de superfícies e volumes de sólidos delimitado por uma superfície gerados por revolução ou gerados a partir de superfícies cilíndricas, considerando como técnica para esboço dessas superfícies o traço da superfície em um plano; constatou também, que para as representações gráficas em três dimensões os livros sugerem a construção em ambientes computacionais, mas não apresentam nenhuma técnica ou esquema de utilização desses ambientes que permitem a realização desse tipo de tarefa.

Para o estudo de funções de várias variáveis, as Integrais Múltiplas são introduzidas como uma continuidade das Integrais Simples. O conceito de representação dos sólidos em sua forma analítica ou em forma gráfica não é discutido embora o registro de representações gráficas esteja presente nos exemplos de exercícios sendo gerados a partir de ambientes computacionais. Assim como para as integrais simples, não apresentam nenhuma técnica ou esquema de utilização desses ambientes que permitem a realização dessas representações.

Quanto aos exercícios propostos, a quantidade é bem abastada, entretanto, exercícios que requerem a articulação entre a representação gráfica e representação analítica dos sólidos envolvidos no estudo das integrais não foram observados. No que se refere ao formalismo matemático, definições e propriedades estão bem estabelecidas, mas existe um empobrecimento significativo quanto à apresentação e demonstrações dos teoremas que mostram que os sólidos estudados são limitados pelas superfícies. Quanto às aplicações das Integrais Duplas em diferentes áreas do conhecimento, essas se limitaram, praticamente, a fenômenos físicos.

Após suas análises documentais aplicou um teste composto por atividades analisadas à luz da Engenharia Didática, Artigue (1998), explorando as Integrais Múltiplas em sua abordagem algébrica e geométrica com exploração da interpretação gráfica. Tal teste foi realizado com os sujeitos de pesquisa brasileiros, divididos em dois grupos, estudantes iniciantes e estudantes avançados - termos usados pelo autor - com o objetivo de verificar como é realizado o ensino dessas integrais, quais as dificuldades apresentadas

pelo objeto do saber no sistema ou praxeologia dos objetos com os quais interage na instituição. 
pelos alunos e como são realizadas as práticas dos professores.

As análises das atividades revelaram que os estudantes apresentaram dificuldades em modelar e interpretar as expressões que eles manipulam vinculadas a esboços das regiões que constroem, principalmente no ambiente lápis e papel; que consideram importante a visualização do sólido para resolução do problema; que "as dificuldades por eles apresentadas podem ser reflexo do ensino atual (pelo menos nas Universidades brasileiras), em que a representação gráfica no espaço não é destacada na organização matemática das Integrais Múltiplas.” (HENRIQUES, 2006, p. 164, tradução nossa).

Posteriormente realizou um estudo das potencialidades do software Maple, de suas restrições e das possíveis dificuldades do seu uso aplicado ao estudo das Integrais Múltiplas no cálculo de medidas de áreas e de volumes de sólidos e, a partir desse estudo, criou uma sequência de operações para o uso do Maple que resultou em uma técnica - conforme definido em Chevallard (1999) - a qual chamou de "Crivo Geométrico" que permite, ao estudante, obter as representações gráficas dos sólidos delimitados por superfícies presentes no ensino das Integrais Múltiplas, propiciando, a ele, estabelecer relações entre a representação gráfica e representação analítica dessas integrais.

O crivo geométrico pode ser entendido como "a definição geométrica do contorno de um sólido" (HENRIQUES, 2007, p. 13). O objetivo explícito dessa técnica é obter um registro gráfico de qualquer sólido isolado ou crivado descrito por várias superfícies. Além disso, ele tem uma importância particular na conversão de registros como proposto em Duval (1993), e consolida a coordenação entre os registros gráfico, analítico e algébrico da integral.

Assim, a partir de suas análises e da criação do crivo geométrico, o autor elaborou o modelo ao qual denominou "MATIM (Modelo para Análise de Tarefas em torno de cálculos de $\mathrm{IM}^{4}$ ) que vincula a análise da resolução de um problema de cálculo de medida de volume por IM à representação gráfica do sólido a ele associado." (HENRIQUES, 2006, p. 219, tradução nossa). Posteriormente, desenvolveu uma nova sequência de atividades que foi aplicada a estudantes em situações de sala de aula ou fora dela, usando como recurso lápis e papel e o ambiente informatizado, analisadas de acordo com o MATIM. As dificuldades apresentadas pelos estudantes relacionadas ao

\footnotetext{
${ }^{4}$ Integrais Múltiplas
} 
estabelecimento das relações entre a representação gráfica e representação analítica dessas integrais, foram, efetivamente, minimizadas no ambiente computacional pelo crivo geométrico, enquanto que no ambiente lápis e papel foram intensificadas pelo grau de dificuldade aumentado das representações gráficas.

Com as análises realizadas, o autor concluiu que a utilização do software na resolução de problemas que envolvem cálculo de áreas e volumes com o uso das Integrais Múltiplas permite um trabalho específico em que a interação entre o usuário e a máquina faz aparecer elementos que articulam a representação gráfica e analítica que favorece o ensino e a aprendizagem desse conteúdo, na medida em que proporcionam a visualização dos elementos gráficos envolvidos na construção do sólido e a compreensão de seus significados relacionados a essas integrais que, muitas vezes, não é proporcionada a partir de construções realizadas com lápis e papel. Finalmente, o autor concluiu que a construção das representações gráficas é fundamental para fazer com que o estudante seja capaz de interpretar, compreender e buscar soluções para os problemas relacionados ao cálculo de volumes, a partir das Integrais Múltiplas.

A tese de doutorado escrita por Miranda (2018), intitulada Esculturas Matemáticas: Atividades para o Estudo da Integral Dupla, teve como objetivo levantar e analisar as concepções de estudantes de engenharia “[...] para o conceito de Integral Dupla que emergem em um cenário para aprendizagem com tarefas ${ }^{5}$ que envolvem a tecnologia e a representação figural ${ }^{6}$ dos sólidos limitados por funções de duas variáveis, por meio das esculturas matemáticas" (p. 19). Os sujeitos de pesquisa foram, inicialmente, estudantes de doutorado da Universidade Anhanguera de São Paulo e posteriormente, alunos de um curso de Engenharia de Produção da Faculdade Flamingo, em São Paulo, em uma situação de curso de extensão.

A pesquisa fundamenta-se, do ponto de vista teórico: na articulação de diferentes abordagens da teoria da Cognição Corporificada, recorrendo às ideias de Lakoff e Johnson (2002), nas questões relacionadas às metáforas conceituais, às ideias de Fauconnier e Tunner (2003), nas situações que envolveram a montagem conceitual no processo da construção de novos conhecimentos, às ideias de Barsalou (2008), principalmente, para a construção das atividades aplicadas aos sujeitos de pesquisa, considerando os processos de simulação por ele discutidos e por utilizar a linguagem

\footnotetext{
${ }^{5}$ Tarefas aqui entendidas como entendido em Cobb, et al., (2004).

${ }^{6}$ Sólidos construídos a partir de uma impressora 3D.
} 
como principal meio para a análise dos discursos.

Os experimentos realizados nessa pesquisa foram organizados de forma a permitir a análise dos discursos dos participantes, bem como, "a evolução desses discursos e as interações com as tarefas e com as esculturas” (MIRANDA, 2018, p. 63). Dessa forma, organizou seu trabalho em dois ciclos, baseados na metodologia Design Experimental apresentada em Cobb et al. (2003):

\section{Ciclo 1:}

Prospectiva

$\checkmark$ Levantamento bibliográfico o que incluiu as pesquisas anteriores (artigos, dissertações e teses); elaboração das tarefas; elaboração do applets, programação dos sólidos para impressora 3D, as esculturas matemáticas.

Reflexiva e prospectiva

$\checkmark$ Implementação do ambiente (CIA) ${ }^{7}$ : o primeiro experimento foi realizado apenas com os applets e organizados em um e-book [...] aplicamos as tarefas em uma turma de Doutorado/Mestrado em Educação Matemática da Universidade Anhanguera de São Paulo, durante as aulas de Didática do Cálculo, cedidas pela professora Dra. Janete Bolite Frant.

Análise Parcial, o que implicou no redesign das tarefas.

Ciclo 2:

Reflexiva e Prospectiva

Implementação do ambiente (CIA): o segundo experimento foi realizando com os applets organizados em um e-book [...] e com as esculturas matemáticas; aplicamos as tarefas em uma turma do Curso de Engenharia de Produção da Faculdade Flamingo, em São Paulo, durante um curso de extensão universitária.

Análise Parcial;

$\checkmark$ Análise Final e criação de uma proposta para o ensino e aprendizagem de Integral Dupla. (MIRANDA, 2018, p.63, 64).

De acordo com a proposta de sua pesquisa, no primeiro ciclo as tarefas foram encaminhadas visando à análise dos discursos dos estudantes e realizadas por meio dos

\footnotetext{
${ }^{7}$ Contexto Interativo de Aprendizagem (CIA).
} 
applets construídos a partir do software GeoGebra 5.0. Exploraram desde a função polinomial de primeiro grau de uma e de duas variáveis até o cálculo de medidas de volumes de sólidos geométricos, por meio das Integrais Duplas, gerados a partir de vários tipos de funções. As tarefas foram aplicadas aos estudantes de doutorado da Universidade Anhanguera de São Paulo aos quais a autora disponibilizou os sólidos geométricos envolvidos nas atividades em impressão 3D, projetados no software OpenScad, adequado à construção desses projetos, a fim de que eles pudessem estabelecer relações entre as propriedades matemáticas existentes nessas formas de representação.

Por meio das análises dos registros de aplicação das atividades dessa tarefa, evidenciaram-se algumas dificuldades apresentadas pelos estudantes durante a sua realização, que apontaram para a necessidade de reorganizá-las. Tais dificuldades estavam relacionadas principalmente à interpretação das questões, a conceitos geométricos não especificados nas questões, às interações com os applets que, por vezes, não permitiram a resolução das integrais, à dificuldade em associar notações gráficas e algébricas de algumas integrais, a não percepção das relações e propriedades que existem entre a representação gráfica das funções de uma e de duas variáveis, bem como a não construção do significados de alguns conteúdos abordados.

Depois de reorganizadas, às atividades da tarefa anterior foi acrescentada uma nova atividade, o processo de integração - nome dado pela autora - na qual foi explorada a ideia geométrica do Teorema de Fubini. A partir daí iniciou-se o segundo ciclo da pesquisa, em que "com o interesse nos discursos dos alunos, nas suas interações sociais e nas manifestações perante as atividades" (MIRANDA, 2018, p. 155), com vistas aos objetivos propostos, as atividades foram aplicadas aos alunos do curso de Engenharia de Produção da Faculdade Flamingo em um ambiente de um curso de extensão universitária.

Nesse ciclo, assim como no primeiro, também foram disponibilizados, aos participantes os sólidos geométricos envolvidos nas atividades, em impressão 3D e os applets criados. Diferentemente do primeiro ciclo, os estudantes realizaram outras atividades referentes à programação computacional no OpenScad. Acompanharam como transformar um arquivo do tipo Cad - Computer Aided Design - para uma plataforma que consegue manipular e mandar as informações para a impressora $3 \mathrm{D}$, por meio do software Repetier-Host C1.5.4 que é de livre acesso, bem como fizeram a impressão 3D 
dos sólidos envolvidos nas atividades da tarefa realizada.

Com as análises do segundo ciclo a autora pode observar que a interação entre os estudantes foi fundamental na realização das tarefas; que eles conseguiram estabelecer comparações entre as representações algébricas e gráficas de funções de uma e duas variáveis; que tais comparações favoreceram à interpretação dessas funções, bem como, de suas formas de representação; que a presença das esculturas matemáticas como uma nova representação para os objetos matemáticos contribuiu para que os estudantes percebessem, mais facilmente, as propriedades matemáticas envolvidas nas representações no espaço mostradas nos applets.

\section{Considerações finais}

Por meio de nossas análises percebemos que Henriques (2006) preocupou-se com aspectos também contemplados na $\mathrm{MCC}$, na medida em que realizou análise de programas institucionais e manuais de referência das instituições, livros didáticos, dentre outros, para entender como as Integrais Múltiplas são abordadas nesses documentos. Também observamos a preocupação do autor em apontar aplicações desse objeto matemático na física e trazer uma discussão em seu texto sobre como essas aplicações estão abordadas nos documentos e livros didáticos analisados. Já no trabalho de Miranda (2018), não identificamos aspectos também contemplados pelos preceitos da MCC.

Tanto Henriques (2006) quanto Miranda (2018) abordam Integrais Múltiplas para cálculo de medidas de áreas de superfícies e de volumes de sólidos, recorrendo à uma contextualização somente na própria matemática, sem buscar o estabelecimento de vínculos entre esse conteúdo e às outras ciências ou entre as áreas de atuação do futuro profissional, o engenheiro, ao contrário do que preconiza a MCC. Não foram encontrados até o momento outros estudos de doutorado tratando dos processos de ensino e de aprendizagem de Integrais Múltiplas, o que aponta para a necessidade de realização de novas pesquisas com essa temática, e, principalmente, que tais pesquisas contemplem vinculações deste objeto matemático com situações de outras áreas do conhecimento presentes nos cursos de engenharia.

\section{Agradecimentos}

Agradeço à CAPES - Coordenação de Aperfeiçoamento de Pessoal de Nível Superior, 
pelo apoio financeiro recebido através da bolsa de doutorado a mim concedida, o que me permitiu a realização desse trabalho.

\section{Referências}

CAMARENA, G. P. El curriculo de las matematicas en ingenieria. In: Memorias de las Mesas redondas sobre definición de líneas de investigación en el ipn. México, D. F.: ipn: p. 21-25. 1984.

. La enseñanza de las matemáticas en el contexto de la ingeniería. In: XXVIII CONGRESO NACIONAL DE LA SOCIEDAD MATEMÁTICA MEXICANA, México. p. 28-35. 1995.

A treinta años de la teoría educativa "Matemática en el Contexto de las Ciencias". Innovación educativa, v. 13, n. 62, p. 17-44, 2013. Recuperado em: http://www.innovacion.ipn.mx/Revistas/Documents/Revistas\%202013/3-A-treintaa\%C3\%B1os-de-la-teor\%C3\%ADa-\%20educativa-62.pdf. Data de acesso: 30 out. 2018.

CAMARENA, G. P; ALLIER, F. Epistemología de lo variacional. In: Memorias del XIII Simposium Internacional: Aportaciones de las Universidades a la Docencia, la Investigación, la Tecnología y el Desarrollo. México. 2012.

CHEVALLARD, Y. Analyse des pratiques enseignantes et didactique des mathematiques: l'approche anthropologique. Recherches em Didactique dês Mathématiques, v. 19, n.2, p.221-26, 1999.

HENRIQUES, A.; ATTIE, J.P.; FARIAS, L. M. S. Referências teóricas da didática francesa: análise didática visando o estudo de integrais múltiplas com auxílio do Maple. Educação Matemática Pesquisa, v.9, n.1, p. 51-81, 2007. Recuperado em: https://revistas.pucsp.br/index.php/emp/article/viewFile/585/436. Data de acesso: 30 out. 2018.

HENRIQUES, AFONSO. L'enseignement et l'apprentissage des intégrales multiples: Analyse didactique intégrant l'usage du logiciel Maple. Mathématiques [math]. Université Joseph-Fourier - Grenoble I, 2006. Français. Recuperado em: https://tel.archives-ouvertes.fr/tel-00100353/document. Data de acesso: 30. out. 2018.

LIMA, G. L.; BIANCHINI, B. L.; GOMES, E. Cálculo e análise: Mapeamento das Pesquisas do GT04 - Educação Matemática no Ensino Superior. Vidya, v. 37, n.2, p. 317-334, jul./dez., 2017. Recuperado em: https://www.periodicos.unifra.br/index.php/VIDYA/article/download/2009/1946. Data de acesso: 30 outubro de 2018.

MIRANDA, G. M. H. Esculturas matemáticas: a atividade para o estudo da integral Dupla. 2018, 321 f. Tese. (Programa de Pós-graduação em Educação Matemática) Universidade Anhanguera. São Paulo, 2018. 